\title{
Analysis and Design on Music Information Management System Based on Android Platform
}

\author{
Xinxin $L v^{1, a}$, Chengwei $\mathrm{Wu}^{2, \mathrm{~b}}$ \\ ${ }^{1}$ College of Education And Sports, Bohai University, Jinzhou, 121013. China \\ ${ }^{2}$ College of Information Science and Technology, Bohai University, Jinzhou, 121013, China \\ ajz_Ivxinxin@163.com, b357889057@qq.com
}

Keywords: music information management; analysis and design; Android platform; data flow diagram; database design; requirement analysis

\begin{abstract}
With the increasingly rapid pace of life in today, mobile devices become more and more popular for people, so the development of a cost-effective information management software has good prospects for development on the Android platform, database design as the basic work it is also particularly important, this paper studied the database design of music information management software, firstly, studied the requirements analysis of music information management software, with data flow diagram describe the course of its operation; Secondly, studied the database design; finally, studied the software security design of music information management software. Database design is good or bad, which directly affects the efficiency of the application and results, therefore, database design of music information management software based on Android platform studied in this paper has great significance for the development of software.
\end{abstract}

\section{Introduction}

As mobile devices become more and more advanced, people's demands have become more sophisticated for mobile devices, from the previous the pursuit of technological to the current pursuit vision, and gradually increase the demands on the system. There are three major mobile operating systems on the market, Windows mobile, Symbian and Android operating system developed Google. Android system which occupies the advantages of open source has the greatest prospects for development [1]. Information management software is one of the applications on the mobile device and attracts people's attention, and the database design of information management software is the foundation of the music information management software development, so the database design of this study has significance. Android is the mobile operating system launched by Google, which is an open mobile development platform in the true sense, is one of the most portable operating system, laid the solid position for Android as the best mobile development platform [2].

Database Design refers to constructing optimal database schema, building databases and their applications for given application environment, enable efficiently store data to meet a variety of application requirements for user. In the database world, use various types of database systems usually referred to database application system [3]. The process of database design includes six stages, namely, the requirement analysis phase, conceptual design stage, the logical structural design phase, the database physical design phase, implementation phase of the database and database operation and maintenance phase [4].

\section{Android Platform Architecture}

Android is divided into four layers, from the top to the lower are the application layer, application framework layer, system runtime layer and Linux core layer, shown in Fig. 1 [5,6].

(1) Application. Applications using the Java language, each application consists of one or more active composition, activities must be based on the Activity class as a super class, the activity is similar to the operating system process, but activity is more flexible than the operating system 
process, and is similar to the process, the activities switch between multiple states. Using Java's cross-platform nature, based on applications of Android framework development cannot compile, running on any computer equipped with the Android system platform, which is the essence of Android.

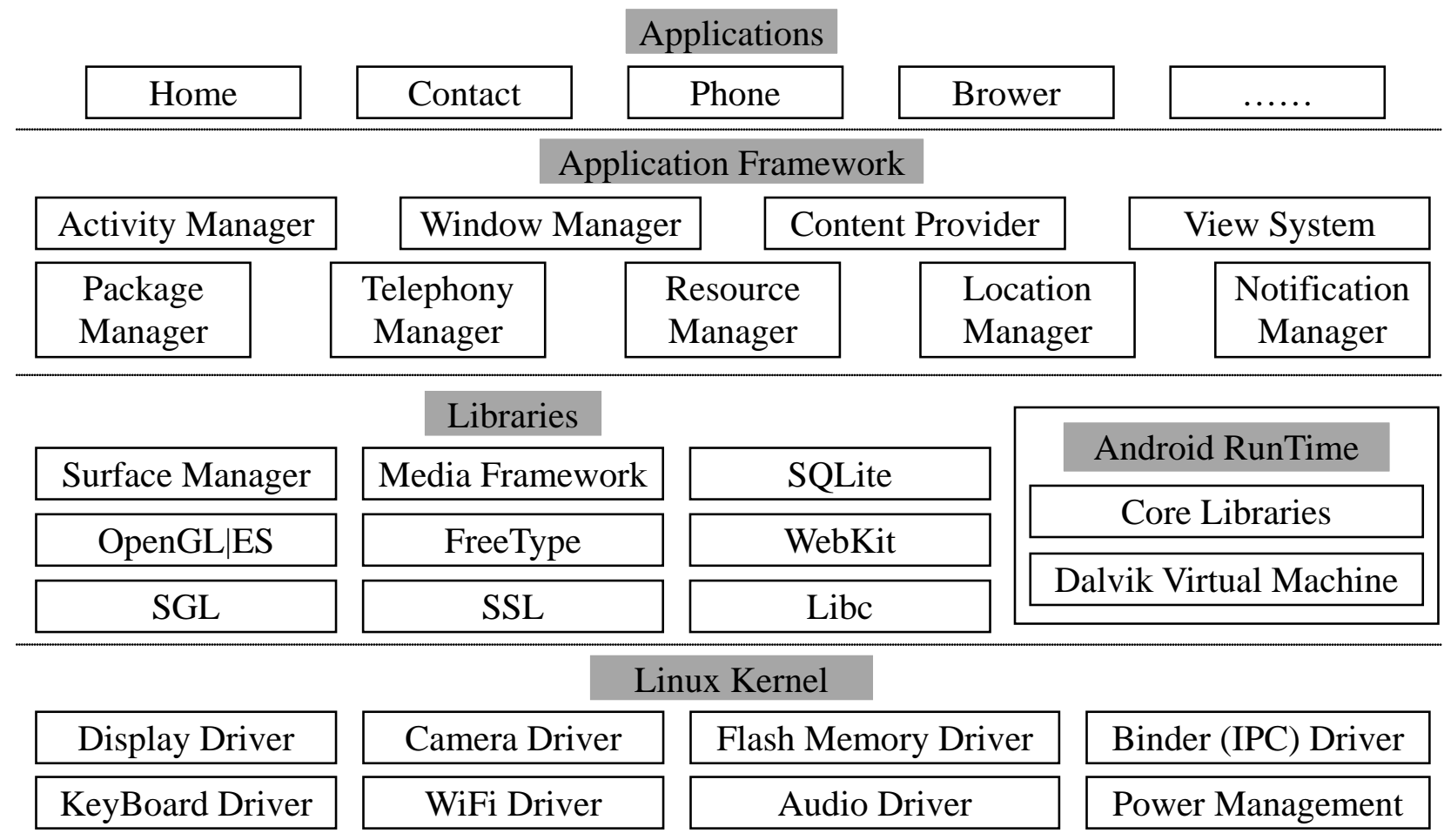

Fig. 1. Android Architecture

(2) Application framework. Android is an open platform, API almost all open to developers. As long as the system application has the function, developers can also provide through the application framework layer provided the API to achieve these functions. For example, the access device hardware information, receives device location information, start the background service and set the alarm clock. Android application architecture to facilitate the reuse of components, any application can release its function modules.

(3) The system runtime layer. It included libraries and Android runtime. Android contains some $\mathrm{C} / \mathrm{C}++$ libraries, used by various components in the Android system, to provide services through the Android application framework for developers. Mainly include the basic C library, multimedia library, bitmap and vector fonts, 2D and 3D graphics engines, browsers, database support. Android's various libraries are generally in the form of system middleware provides.

(4) Linux kernel layer. The previous Android relies on Linux2.6 kernel, the latest Android4.0 series relies on the latest Linux 3.x kernel. Series of the Linux kernel provides Android system the basic network protocol stack, driving model, security, memory management, process management function.

\section{Requirement Analysis}

In database design, requirements analysis refers to detailed analyzing the problem to be solved, to clarify the requirements of issue, analysis and survey user's business activity and the usage of data, to determine the user requirements and constraints for the database system, and ultimately to form requirements specification. It can be say that requirements analysis is necessary work early in the software development, accurately understanding and analyzing user requirements (including data and processing) is the basis for the entire design process, determines the success or failure of software development work, is the most difficult and time-consuming step [7]. Requirements analysis commonly used some methods including structured approach, object-oriented methods, prototyping, and use case modeling and so on. 
Data flow diagrams are the result generated in the requirements analysis phase, using it to describe the specific workflow for software. Data flow diagram (DFD) is a graphics technology, which describes the information flow and when the data from the input moves to output is applied, it is a kind of functional model. Data flow diagrams have four basic components, which are processing, data source / end point, data stored and data flow [8]. There are two typical structure in the data flow graph, one is inverter type structure, expressed work can be described the input, main processing and output, it is linear state. Another is the transaction structure; this data flow diagram was bundle, namely a bunch of data streams parallel input or output, there are several transactions may require treatment. Step of the data flow diagram is divided into three areas:

(1) Determine the input and output, namely top chart;

(2) Design of the internal system, namely the underlying data flow diagrams, using the principle that top-down, outside-inside;

(3) Pay attention to some issues, such as name, data flow, branch balancing, local data storage.

According to the theory and the steps of the data flow diagram, the data flow diagram of music information management software is shown in Fig. 2.

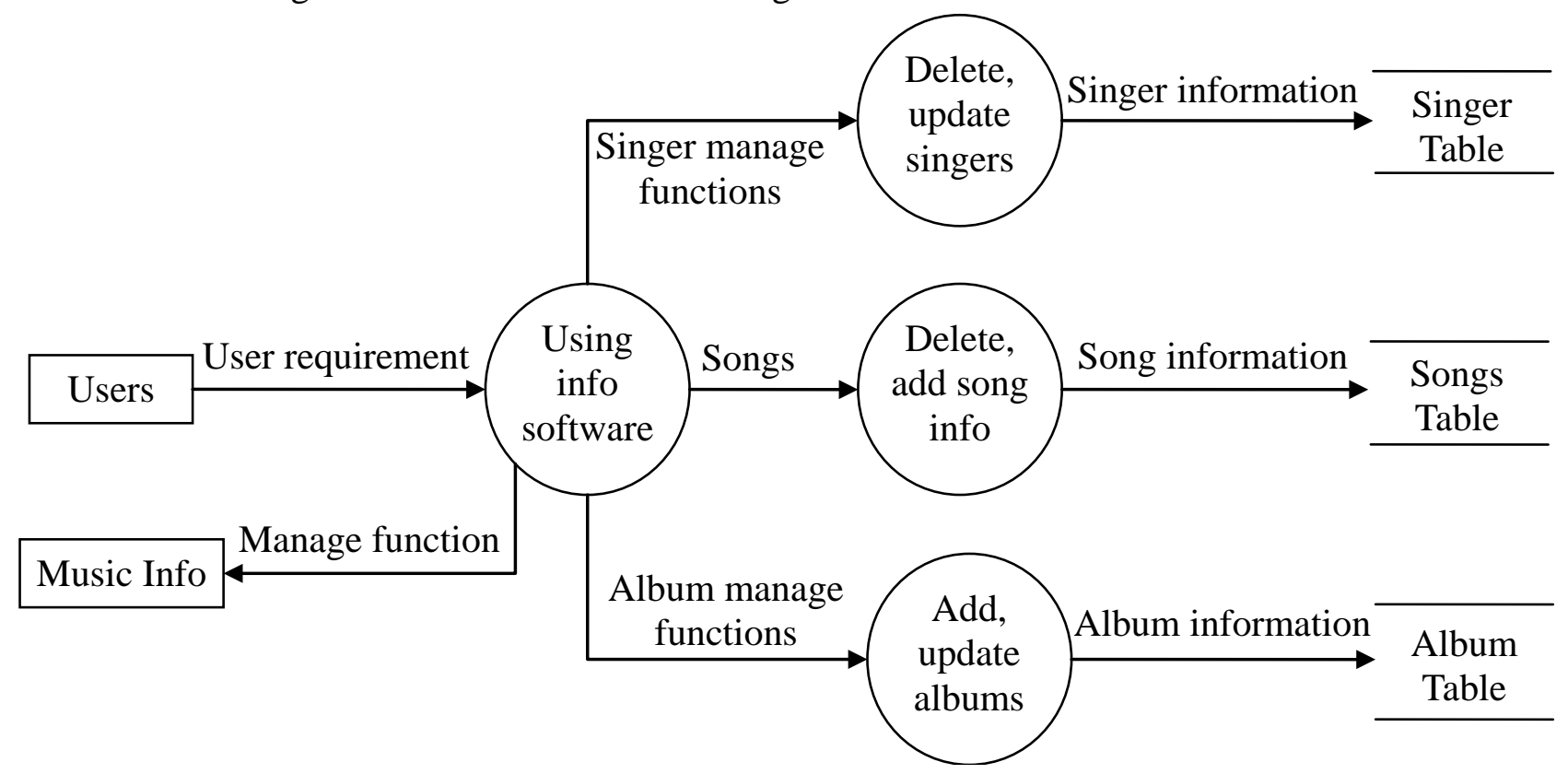

Fig. 2. Data flow diagram on music information management software

\section{Database Design}

Database design studied in this paper is based on MySQL database, MySQL is a multi-threaded SQL (Structured Query Language) Server, is a true multi-user servers. MySQL consists of a server daemon mysqld and many different client programs and libraries, based on the structure of the client / server to achieve. Solid, fast, and easy to use are the main goals for MySQL [9].

According to the requirement analysis of music information management software to design database, to create a lot of table structures in the database of music information management software, including songs tables, singer table, song annex table, songwriter table, album table, singer type table, song type table and so on. Song table mainly store song information, including song number, song title, singer number, lyrics, lyricist number, album number, etc.; singer table mainly store singer information, including singer number, singer name, singer type, sex, age, citizenship, affiliated company, singer rankings, album quantity and so on; songs annex table shows the relationship between the singers table and songs table, including songs number, singers number, store address; songwriter table stores the information of songwriter, mainly including lyricist number, lyricist name, sex, representative, etc.; album table main store album information, including album number, album name, tracks quantity, release date, release location, lyricist number, album ranking and album introduction; singer type table mainly store singer types, such as 
creative type, rock type, etc., properties include the type number, type name, type description; song type table stores songs type, such as classical, pop, jazz, religion and so on.

The local structural diagram of music information management software based on Android is shown in Fig. 3.

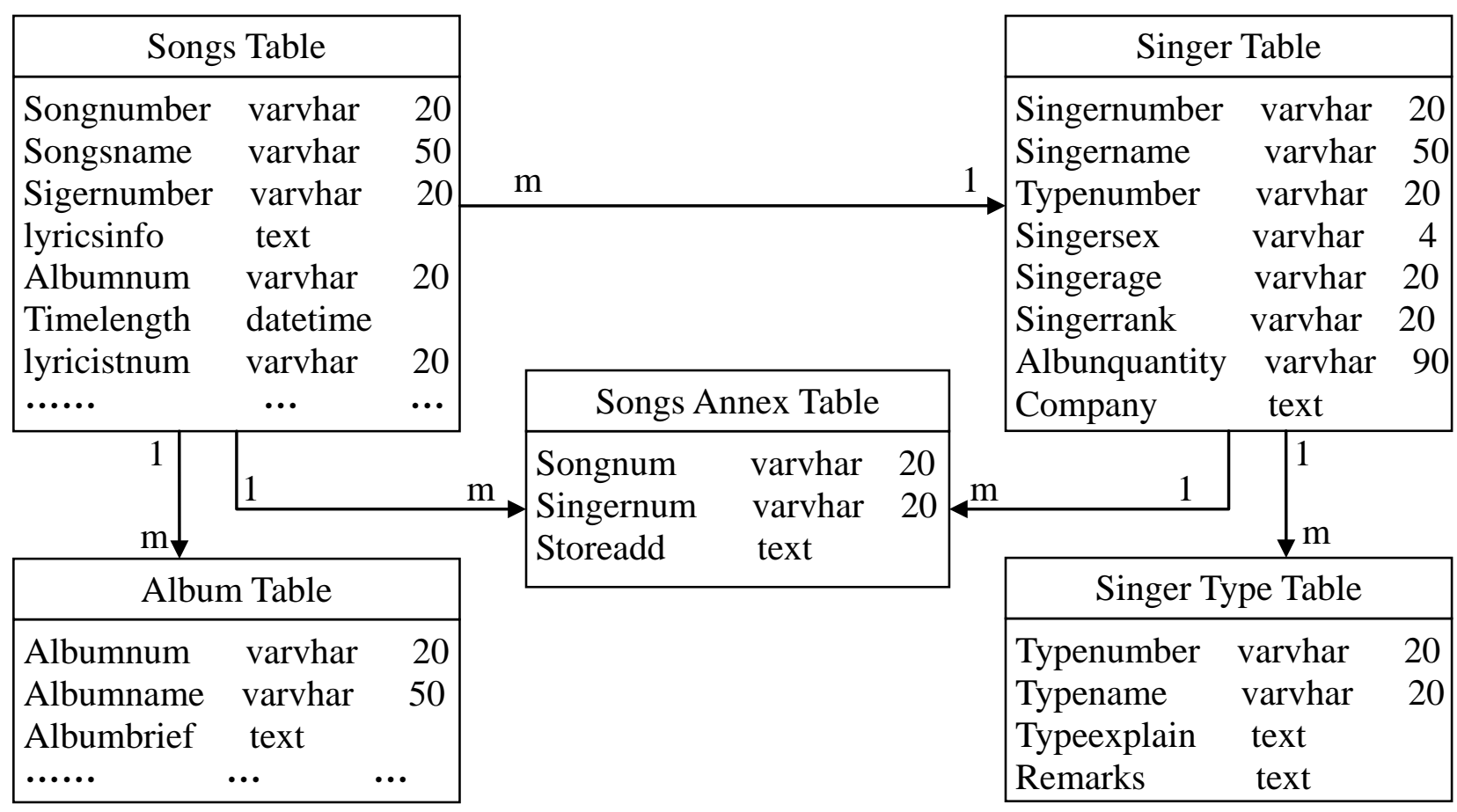

Fig. 3. Local structural diagram of music information management software

\section{Security Design}

Security of the database refers to protecting databases to prevent data leakage, alteration or destruction caused by illegal using. The basic requirements include access control, data exclude camouflage, user authentication and reliability. Mainly consider two aspects, one is from the operating system and network security, and the other is the safety of the database itself. The main safety technology on the operating system platform has operating system security policy, security management strategy, data security and other aspects. Effective measures taken by the network include: firewall, intrusion detection, etc. Database own security includes the security of database management systems, database encryption, data backup and restore, and databases hierarchical control [10].

The security design of Android system ideologically inherited the Linux design ideas, based on the Linux kernel, Android offers a variety of core services such as security, memory management, process management, network management, driver model, etc.; data security mainly relies on software signatures mechanisms to guarantee. Android and applications need to be signed; when publishing it must firstly generate a public key and a private key through the development / tools / make_key. Android system security mainly includes the following aspects: the kernel exist many loopholes, lack of virus protection, and lack of security audits and regulatory as well as software development kits exists many risks [11].

Database security enhancement program includes [12]: (1) user identification and authentication, application or database users provides own valid proof of identity, only legal status can access the database, the user ID is the premise of database safety; (2) access control, the purpose of access control is to ensure that users only can do authorized operation; (3) database encryption, it prevents unauthorized users using loopholes existed in the system to steal database file or tamper the database contents; (4) database operations audit, it is a security mechanism which monitor and 
record all operations user or application does for database, the function of auditing is stored in the audit log for later analysis.

\section{Conclusion}

With the extensive use of computer applications and the rapid development of the mobile phone market, a variety of audio resources are widely circulated online, these resources appear to be normal, but has gradually become an indispensable part of life. Nowadays life is stressful, listening to music is one of the best ways to relieve stress, for convenience, people like to use a mobile device to play music, and so for the multimedia software on mobile devices, the music information management is also important [13]. Therefore, in order to meet people's needs to design music information management software, this paper mainly studies the database design of the music information management software. Described the analysis method and analyzed the user needs for the music information management software, and then build a database, and finally consider the safety performance, in line with the idea of database design [14]. Database design affects the entire application development process, is the basis for software development, so based on the Android platform, database design of music information management software affects not only music software development, but also more skilled Android technology and other features on the market for designers.

\section{Acknowledgement}

This work is supported by general subject of "the Twelfth Five-year Plan" on educational science of Liaoning province: Current situation analysis and countermeasure research on rural primary and middle school basic music education of Liaoning province, 2014, Approval (JG14CB005).

\section{References}

[1] G. H. Sheng, "Mobile Platform Music Player Analysis Report," Kownledge Economy, vol. 14, no. 24, pp. 99, 2012.

[2] Q. Y. Zhang, "Design and Implementation of Intelligent Music Player Based on Android Platform," Computer CD Software and Applications, vol. 16, no. 14, pp. 49-50, 2013.

[3] H. C. Li, Y. Guo, H. G. Dong, "Analysis of Database Design," Digital Technology \& Application, vol. 18, no. 8, pp. 176, 2010.

[4] Baidu Baike, "namespace," http://baike.baidu.com/view/8268.htm?fr=aladdin, 2014-9-22.

[5] Hui Zhao, Min Chen, Meikang Qiu, et al, "A novel pre-cache schema for high performance Android system," Future Generation Computer Systems, vol. 56, no. 3, pp. 766-772, 2016.

[6] Cynthia\&Sky, "The basic structure of Android," http://www.cnblogs.com/lijunamneg/archive/2013/01/18/2866953.html, 2015-12-30.

[7] S. Wang, X. S. Sa, "Introduction to Database Systems," Higher Education Press, 2005.

[8] L. Bai, Q. X. Ling, "Application of the Data Flow Diagram in the Information Processing," Electro- Optic Techonology Application, vol. 20, no. 6, pp. 65-67, 2005.

[9] R. Y. Ling, "Android-based Student Information Management System Design and Implementation," Master's Degree of University of Electronic Science and Technology, 2012.

[10] Z. Liu, D. S. Yu, "Database Security Analysis," China Computer\&Communication, vol. 5, no. 2, pp. 99, 2011.

[11] Q. Y. Chen, "Analysis of the Safety Performance of the Android System," China CIO News, vol. 14, no. 9, pp. 60-62, 2011.

[12] L. X. Huang, "Research of Data Protection Security Technology on the Android System," Master's Degree of Nanjing University of Technology and Engineering, 2014. [13] Baidu
http://wenku.baidu.com/view/a7522d2b2af90242a895e566.html?re=view, 2014-9-22. 
[14] L. Zhang, L. Ma, "Discussionon Database Design," Journal of Anyang Institute ofTechnology, vol. 6, no. 4, pp. 76-79, 2007. 